\title{
Synthetically Interpolated Five-minute Direct Normal Irradiance
}

\author{
$\underline{\text { A. P. Grantham }}^{\text {a }}$, P. J. Pudney ${ }^{\text {a }}$, J. W. Boland ${ }^{\text {a }}$, M. Belusko ${ }^{\text {a }}$ \\ ${ }^{a}$ School of Information Technology and Mathematical Sciences, University of South Australia, GPO BOX \\ 2471, Adelaide, South Australia, 5000 \\ Email: adrian.grantham@unisa.edu.au
}

\begin{abstract}
The electricity generated from Concentrated Solar Thermal (CST) systems is mostly influenced by the amount of Direct Normal Irradiance (DNI). The majority of DNI values found in both historical and Typical Meteorological Year (TMY) data are hourly means. However, hourly data is too infrequent to capture the transient effects of clouds. High temporal resolution DNI is required to capture these effects on DNI and subsequent influences on CST output.

This paper demonstrates a method where five-minute DNI data from one location is used to develop synthetic variations in the hourly data from another location.

We use five-minute DNI from Adelaide, South Australia, where real five-minute data is available. Five-minute clear sky ratios (ratio of observed DNI to modelled perfect clear sky DNI) are calculated for each 5-minute time period. Hourly clear sky ratios are calculated for each hourly time period. The hourly ratio identifies a clear sky bin into which the twelve five-minute ratios corresponding to the hourly ratio are placed. The result is clear sky indexes each containing five-minute ratios that capture how five-minute DNI fluctuates for each clear sky index.
\end{abstract}

The process for generating synthetically interpolated five-minute DNI from hourly DNI is straightforward. Hourly clear sky ratios for each hourly time period are calculated to identify the clear sky index from which 12 five-minute synthetic clear sky ratios are bootstrapped (resampled with replacement) for each hour. The synthetic five-minute ratios are multiplied by the five-minute modelled perfect clear sky DNI to give synthetically interpolated five-minute DNI.

Comparisons between synthetically generated five-minute DNI and observed five-minute DNI are made for Adelaide to determine the performance of this method. A mean bias deviation (MBD) of $-0.4 \%$ and a normalised root mean square deviation (NRMSD) of $16.3 \%$ compares favourably with other methods described in the literature, which have a MBD of $0.3 \%$ and NRMSD of $31 \%$. The level of variation in the synthetic compares well to the observed with standard deviations of 390.0 and 391.3 respectively.

Of particular interest is how well the model performs when applied to hourly mean DNI for Woomera South Australia. A MBD of $-1 \%$, a NRMSD of $15 \%$ and standard deviations of 373.5 and 372.4 , for the synthetic and observed five-minute DNI respectively, indicate the method is also performing well for Woomera. It is important to note the synthetic DNI for Woomera is derived from a model based on five-minute DNI from Adelaide.

This is an important result because it means the method could also be applied to other locations where only hourly DNI exists. Therefore, not only is the temporal resolution of DNI extended to five-minutes, but the spatial coverage of five-minute DNI is also extended. These results are important in determining the role solar energy will play in a $100 \%$ renewable energy system.

Keywords: Direct Normal Irradiance, DNI, solar radiation, interpolated 


\section{INTRODUCTION}

The electricity generated from Concentrated Solar Thermal (CST) systems is mostly influenced by the amount of Direct Normal Irradiance (DNI), which compared to global irradiance, exhibits greater variability (Meyer et al., 2009). The physical phenomenon explaining this is the transient effects of clouds.

The majority of DNI found in historical and Typical Meteorological Year (TMY) data are hourly means. However, hourly measurements are too infrequent to capture the transient effects of clouds. Solar energy can vary significantly over an hour. Gansler et al. (1995) (cited in Assunção et al. (2007)) found that the "cumulative distribution function of solar radiation quantities measured during one minute-interval (instantaneous values) differs considerably from ones measured during one hour-interval".

This raises the question of what is the optimal temporal resolution. Suehrcke and McCormick (1988) (cited in Assunção et al. (2007)) observed that the frequency distribution of five-minute (averaged solar irradiance) values is similar to the frequency distribution of instantaneous values. They also concluded that the statistical properties of instantaneous solar irradiance values are not represented properly by hourly or daily values.

From a renewable energy market operator's perspective, hourly DNI does not provide the necessary detail required to design and operate a renewable energy system containing CST systems. Because five-minute DNI captures more of the variation inherent with solar energy, operators are given more insight and therefore can provide a more robust and reliable energy system compared to a system using hourly DNI.

Unfortunately five-minute DNI is spatially sparse compared to hourly data.

Therefore, the purpose of this research is to develop a method for generating synthetically interpolated fiveminute DNI that can be applied to multiple locations, where only hourly DNI exists, to provide both high resolution temporal and spatial DNI.

\section{DATA}

The DNI datasets are split into three groups; observed five-minute DNI, observed hourly mean DNI and modelled perfect clear sky DNI.

The DNI data from which the synthetically interpolated five-minute DNI model is derived, consists of observed five-minute DNI for Adelaide, South Australia, from March 2003 to October 2012. Only five-minute DNI belonging to a complete day is included resulting in 3,099 days of data. February 29 days were excluded.

The DNI data to which the synthetically interpolated five-minute model is applied, consists of hourly mean DNI observations for Adelaide for the same period. The model is also applied to hourly mean observations for Woomera, South Australia, located approximately $450 \mathrm{~km}$ north, north west of Adelaide. The Woomera data is for a time period from April 2012 to March 2013. Once again only DNI belonging to a complete day is included resulting in 328 days of hourly data. The purpose of using Woomera data is to test how suitable the model (derived from Adelaide five-minute data) can be applied to other locations.

The synthetically interpolated five-minute DNI is compared against the five-minute DNI observations for Adelaide and Woomera for the same time periods.

Modelled perfect clear sky data consists of five-minute and hourly mean perfect clear sky DNI for one year for both Adelaide and Woomera. Perfect clear sky DNI was modelling using equations from Boxel (2002).

All observed DNI data was obtained from the Australian Bureau of Meteorology website (Bureau of Meteorology, 2013). The DNI data was originally in one-minute time intervals but was downscaled to five-minute and hourly time intervals by taking the average of the one-minute data for each five-minute and hourly period.

Each five-minute interval is delineated in line with every five minutes in time, ranging from 06:01 to 06:05, with five-minute time stamps at 06:05, 06:10, etc. Hourly intervals are analogous to this with time stamps at the top of the hour, i.e. 06:00, 07:00, etc.

\section{NOMENCLATURE}

We use the following definitions:

- $I_{i, j}$ is the observed five-minute DNI during hour $\mathrm{i}$ and five-minute period $\mathrm{j}$.

- $\bar{I}_{i}=\frac{\sum_{j=1}^{12} I_{i, j}}{12}$ is the observed hourly mean DNI for hour $\mathrm{i}$.

- $c_{i, j}$ is the modelled perfect clear sky five-minute DNI during hour i and five-minute period $\mathrm{j}$, given by equations in Boxel (2002) assuming a turbidity factor of 2.4 for Adelaide and 2.5 for Woomera.

- $\bar{c}_{i}=\frac{\sum_{j=1}^{12} c_{i, j}}{12}$ is the modelled perfect clear sky hourly mean DNI for hour $\mathrm{i}$.

- $R_{i}=\frac{\bar{I}_{i}}{\bar{c}_{i}}$ is the hourly clear sky ratio. 
A. P. Grantham et al., Synthetically Interpolated Five-minute Direct Normal Irradiance

- $R_{\max }$ is the maximum daily $R_{i}$.

- $a_{i, j}=\left\{\begin{array}{ll}c_{i, j} \times R_{\max } & \text { if } R_{\max }>0.9 \\ c_{i, j} & \text { if } R_{\max } \leq 0.9\end{array}\right.$ is the adjusted modelled perfect clear sky five-minute DNI during hour $\mathrm{i}$ and five-minute period $\mathrm{j}$.

- $\bar{a}_{i}=\left\{\begin{array}{ll}\bar{c}_{i} \times R_{\max } & \text { if } R_{\max }>0.9 \\ \bar{c}_{i} & \text { if } R_{\max } \leq 0.9\end{array}\right.$ is the adjusted modelled perfect clear sky hourly mean DNI for hour $\mathrm{i}$.

- $R_{i, j}^{*}=\frac{I_{i, j}}{a_{i, j}}$ is the adjusted five-minute clear sky ratio during hour $\mathrm{i}$ and five-minute period $\mathrm{j}$.

- $R_{i}^{*}=\frac{\bar{I}_{i}}{\bar{a}_{i}}$ is the adjusted hourly clear sky ratio.

- $b_{R}=\left\lfloor 100 \times R_{i}^{*}\right\rceil$ is the clear sky bin for hourly ratio $R_{i}^{*}$.

- $\tilde{I}_{i, j}$ is the synthetically interpolated five-minute DNI during hour $\mathrm{i}$ and five-minute period $\mathrm{j}$.

The transmittance and turbidity of the atmosphere can vary from day to day even without clouds. We make adjustments to the modelled perfect clear sky DNI to deal with this effect. Figure 1 shows two days of observed five-minute and modelled perfect clear sky DNI. The observed DNI in Figures 1(a) and 1(b) both depict clear sky days with no cloud. Yet the modelled perfect clear sky DNI for day two clearly overestimates the DNI, and should be adjusted.

To adjust the clear sky model, we calculate the hourly ratios, $R_{i}$, for each day. If the maximum $R_{i}$ is greater than 0.9 for any hour then we assume that the sky was clear during that hour and scale our clear sky model for the day to match the observed hourly DNI during that hour. The results of this scaling are shown in Figures $1(\mathrm{c})$ and $1(\mathrm{~d})$.

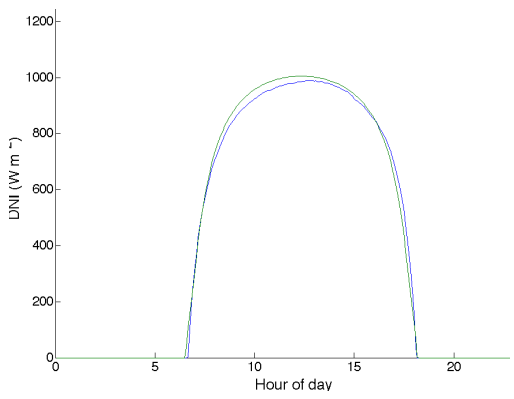

(a) Clear sky One

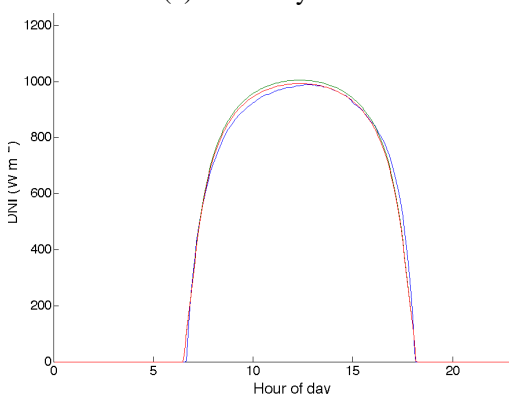

(c) Adjusted clear sky One

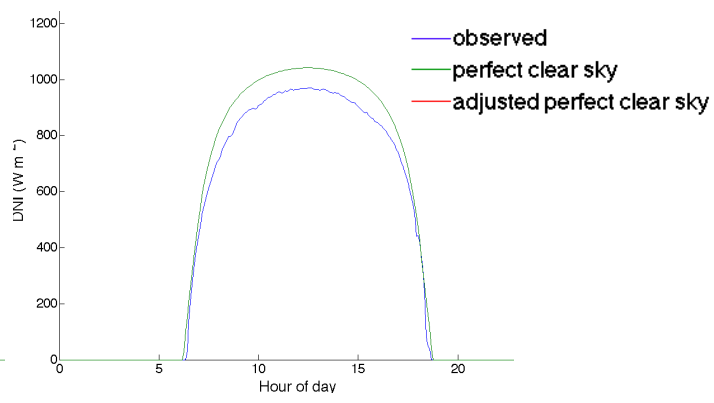

(b) Clear sky Two

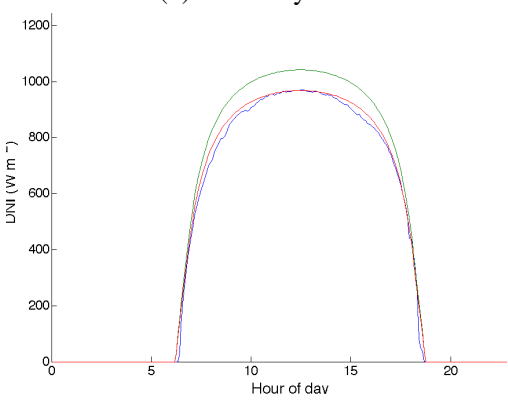

(d) Adjusted clear sky Two

Figure 1. Different degrees of clear sky days

The desired effect of this adjustment ensures the daily five-minute ratios are categorised consistently irrespective of the daily variation in transmittance or turbidity.

It was determined that a ratio of 0.9 was an appropriate threshold of clear sky because of its positive effect on results, both visually and statistically, compared to other ratios.

\section{METHODOLOGY}

\subsection{Background}

Polo et al. (2011) proposes a method to generate synthetic solar irradiance (global horizontal and DNI) at tenminute intervals from hourly data by adding the contributions from the hourly mean to a stochastic fluctuation about the mean. Contributions from the hourly mean are derived from the cubic interpolation of the hourly values for the ten-minute intervals. This ensures the ten-minute data has a similar shape to the hourly data. 
The stochastic contribution accounts for the transient effects of clouds and is modelled by first calculating the normalised standard deviations (standard deviations divided by the maximum standard deviation) of the tenminute data for each hour. The normalised standard deviations are then divided into four sky condition stages according to each value's normalised clearness index. Then beta distributions are fitted to each sky condition.

Stochastic fluctuations are then added by firstly calculating the normalised clearness index for the hourly value. The next step is to randomly generate beta distributed normalised standard deviations from the sky condition stage according to the hourly normalised clearness index. The normalised standard deviations are then denormalised to obtain an amplitude of the fluctuation. The amplitude is then randomly added or subtracted to the interpolated hourly irradiance value.

Our method for generating synthetic DNI is similar. However:

- Instead of the normalised clearness index, we use clear sky ratios to categorise different sky conditions. The clear sky ratio is a ratio of observed DNI at the Earth's surface to modelled perfect clear sky DNI at the Earth's surface. A ratio of 1 indicates a perfect clear sky whilst a ratio of 0 indicates complete cloud cover blocking the direct beam from the sun.

- Clear sky conditions are divided into ratio increments of 0.01 instead of four clear sky conditions. This fine level of categorisation provides a near continuous approach to generating synthetic five-minute DNI. Instead of fitting beta distributions to each category, the technique of bootstrapping (resampling with replacement) is used (Efron, 1992). This technique is justified because the minimum number of five-minute observations in each of the categories is 816 which is large enough.

- The method presented here incorporates modelled perfect clear sky DNI, which captures the daily shape of DNI. Therefore, cubic interpolations are not necessary.

\subsection{Method}

Developing the Model. The model is developed using observed five-minute DNI from Adelaide. For each day:

1. Calculate $R_{i}$ for all daytime hours (absolute zenith angle less than 90 degrees) except for the first two hours after sunrise and the last two hours before sunset. The morning and evening hours are not included so as to not include any of the irregularities that can occur with DNI during sunrise and sunset periods.

2. Find $R_{\max }$.

3. Adjust $a_{i, j}$ and $\bar{a}_{i}$ according to $R_{\max }$.

4. Calculate $R_{i, j}^{*}$ and $R_{i}^{*}$.

5. Place the twelve $R_{i, j}^{*}$ five-minute ratios for each hour into bin $b_{R}$ according to hourly ratio $R_{i}^{*}$.

The modelling process results in clear sky indexes containing five-minute ratios that capture how five-minute DNI fluctuates during the corresponding clear sky hour.

Generating Synthetically Interpolated Five-minute DNI. Synthetically interpolated five-minute DNI is generated based on hourly mean DNI for Adelaide and Woomera. For each day:

1. Calculate $R_{i}$ for all daytime hours, ignoring the first two hours after sunrise and the last two hours before sunset.

2. Find $R_{\max }$.

3. Adjust $a_{i, j}$ and $\bar{a}_{i}$ according to $R_{\max }$.

4. Calculate $R_{i}^{*}$ but include the first two hours after sunrise and the last two hours before sunset. All daytime hourly ratios are now required in order to generate the synthetic five-minute DNI from sunrise to sunset.

5. Determine bin $b_{R}$ for each hour. If the bin is empty, step down in bin in 0.01 increments until a nonempty bin is found (stepping down in bin was not necessary for Adelaide and was only necessary for Woomera for the hourly clear sky ratio of 1.01).

6 . For each hour bootstrap (resample with replacement) twelve synthetic five-minute ratios, $R_{i, j}^{*}$, from bin $b_{R}$.

7. Calculate synthetically interpolated five-minute DNI, $\tilde{I}_{i, j}=R_{i, j}^{*} \times a_{i, j}$.

The method automatically generates zero DNI at night, and cannot generate negative DNI values. 


\section{RESULTS}

Synthetically generated five-minute DNI was generated for Adelaide and Woomera based on hourly ratios. It is important to note the five-minute synthetic generation for Woomera is derived from applying the clear sky indexed five-minute data from Adelaide.

Figures 2 and 3 show example days of synthetically interpolated five-minute DNI compared to the observed DNI for the same day for Adelaide and Woomera respectively.

For complete clear sky days in figures 2(a), 2(b), 3(a) and 3(b) the synthetic DNI is performing very well compared to the observed DNI. The synthetic is capturing the overall shape of the observed and is doing a very good job at matching the observed point to point.

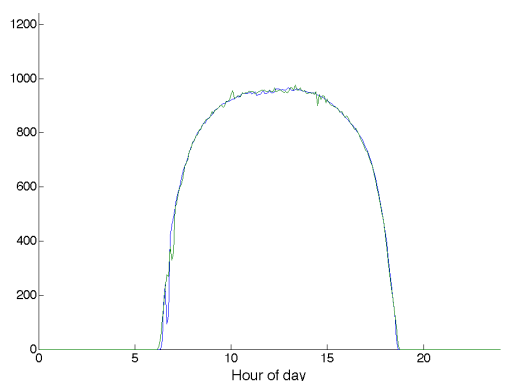

(a) Adelaide clear sky

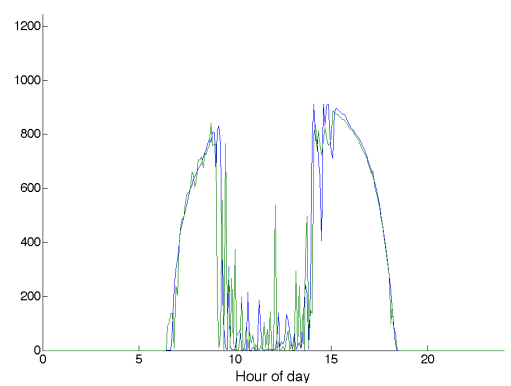

(d) Adelaide partly cloudy sky

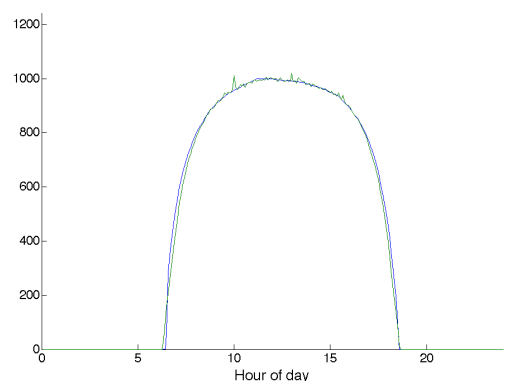

(b) Adelaide clear sky

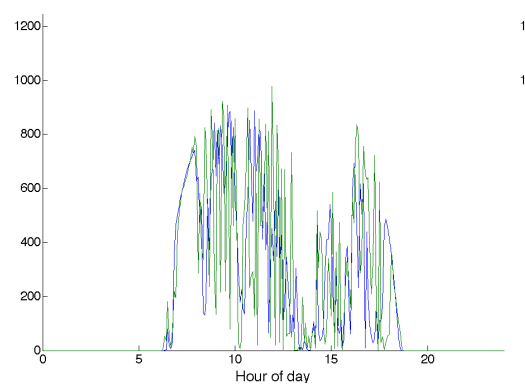

(e) Adelaide cloudy sky

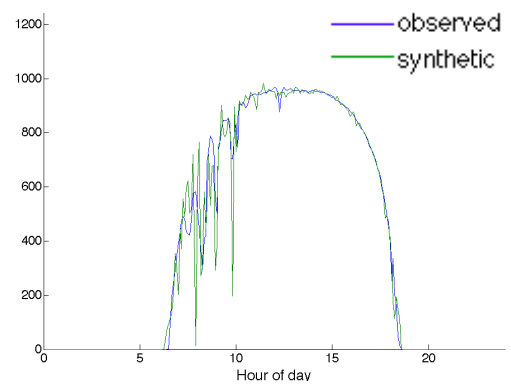

(c) Adelaide partly cloudy sky

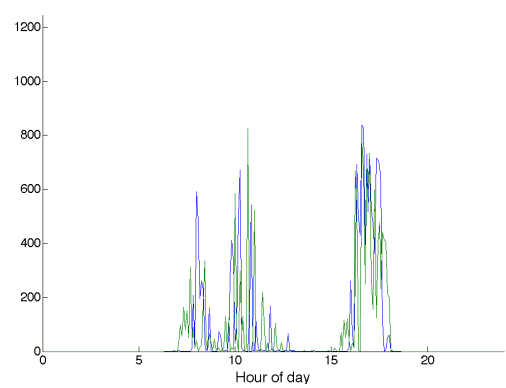

(f) Adelaide cloudy sky

Figure 2. Adelaide five-minute synthetic vs observed DNI

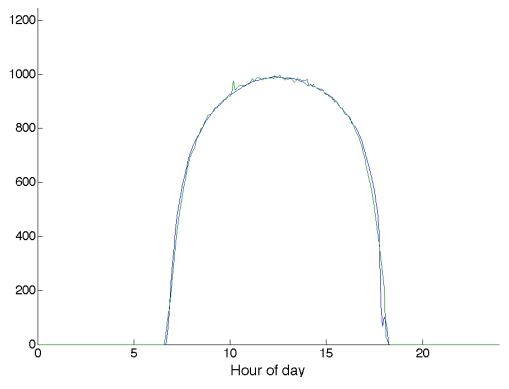

(a) Woomera clear sky

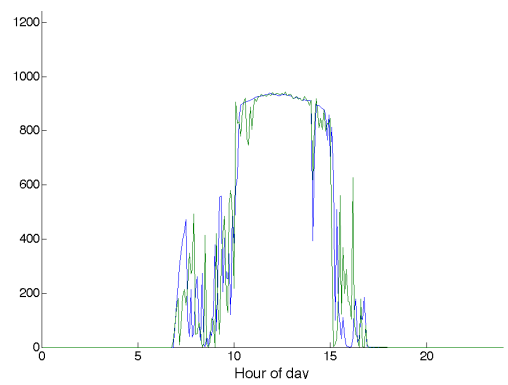

(d) Woomera partly cloudy sky

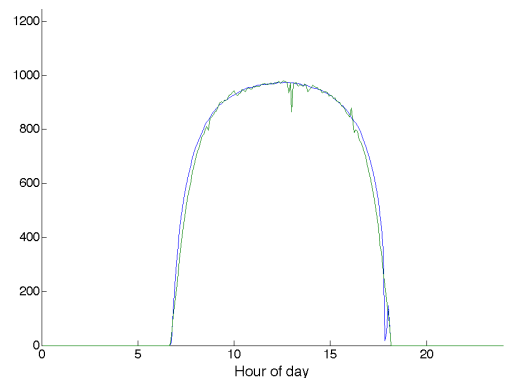

(b) Woomera clear sky

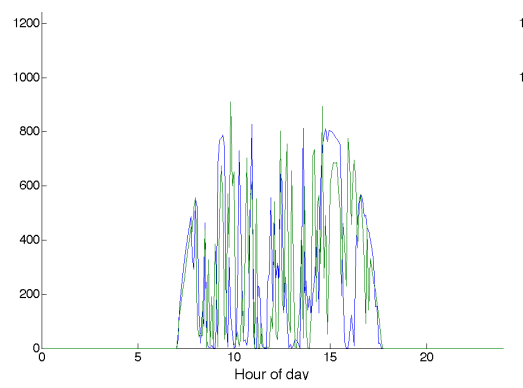

(e) Woomera cloudy sky

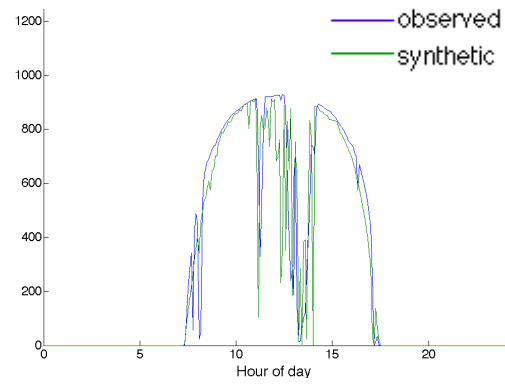

(c) Woomera partly cloudy sky

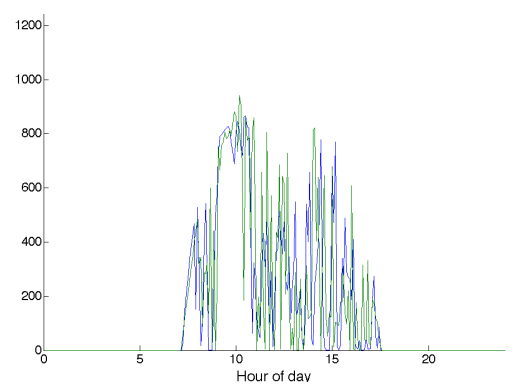

(f) Woomera cloudy sky

Figure 3. Woomera five-minute synthetic vs observed DNI

Figures 2(c), 2(d), 3(c) and 3(d) show examples of partly cloudy days. During the clear sky periods of the day, the synthetic performs just like it did for complete clear sky days. During the cloudy periods of the day, 
the synthetic is not matching the observed DNI point to point. However, that is not the aim here. Recall the synthetic five-minute DNI is based only on hourly DNI - there is no way of knowing what the real DNI values were for each five-minute period. Instead, the aim is to firstly capture the overall shape, secondly match the degree of variation (not necessarily the exact variation) and thirdly, ensure the long term statistics of the synthetic match those of the observed. For the cloudy periods here, the synthetic appears to be capturing the variation exhibited in the observed data.

Examples of cloudy days are shown in figures 2(e) and 2(f). Once again the aim is not to match point to point but capture the shape and variation of the observed, which the synthetic data is doing here.

Normalised root mean square deviation (NRMSD) and mean bias deviation (MBD) statistical measures are shown in Table 1. The MBD and NRMSD are calculated using Equations (1) and (2) where $\hat{y}_{i}$ is the modelled value for time period $\mathrm{i}, y_{i}$ is the observed value for time period $\mathrm{i}, y_{\max }$ is the maximum value of the observed, $y_{\min }$ is the minimum value of the observed and $\mathrm{n}$ is the number of values.

\begin{tabular}{|c|c|c|c|}
\hline$M B D=\frac{\frac{1}{n} \sum_{i=1}^{n}\left(\hat{y}_{i}-y_{i}\right)}{\frac{1}{n} \sum_{i=1}^{n}\left(y_{i}\right)}$ & (1) & $N R M S D$ & $\frac{\sqrt{\frac{\sum_{i=1}^{n}\left(\hat{y}_{i}-y_{i}\right)^{2}}{n}}}{y_{\max }-y_{\min }}$ \\
\hline Tab & 1. Statist & & \\
\hline & $\begin{array}{c}\text { Adelaide } \\
\%\end{array}$ & $\begin{array}{c}\text { Woomera } \\
\%\end{array}$ & $\begin{array}{c}\text { Polo et al. (2011) } \\
\%\end{array}$ \\
\hline 5-minute MBD & -0.4 & -1 & 0.3 \\
\hline 5-minute NRMSD & 16.3 & 15 & 31 \\
\hline 5-minute MBD at 09:00 & -2.8 & -1.23 & - \\
\hline 5-minute MBD at 12:00 & -1.18 & 1.61 & - \\
\hline 5-minute MBD at 15:00 & 0.33 & 2.57 & - \\
\hline Hourly irradiation totals MBD & -0.3 & -1 & - \\
\hline Hourly irradiation totals NRMSD & 12.8 & 14.2 & - \\
\hline Daily irradiation totals MBD & -0.4 & -1 & - \\
\hline Daily irradiation totals NRMSD & 1 & 1.2 & $2-4$ \\
\hline Daily standard deviations MBD & -0.05 & 1.7 & - \\
\hline Daily standard deviations NRMSD & 4.2 & 4.4 & - \\
\hline
\end{tabular}

A MBD of $-0.4 \%$ and a NRMSD of $16.3 \%$ of the five-minute DNI for Adelaide indicates that the synthetic is performing well, albeit slightly underestimating. This compares favourably with Polo et al. (2011) which reports a MBD of $0.3 \%$ and NRMSD of $31 \%$. This could be because the method presented here uses clear sky conditions in ratio increments of 0.01 , providing a more continuous approach to generating synthetic DNI, whereas Polo et al. (2011) uses only four clear sky conditions.

However, the bias at specific times during the day (09:00, 12:00 and 15:00) varies. A MBD of -2.8\% at 09:00 and $0.33 \%$ at 15:00 for Adelaide might suggest clouds behave differently in the morning to the afternoon. The reason for this could be the method presented here categorises clear sky ratios irrespective of time of day.

Because the method generates synthetic DNI on five-minute time scales, it is more practical to examine the MBD and NRMSD for the hourly and daily irradiation totals. An hourly MBD of $-0.3 \%$ and NRMSD of $12.6 \%$ and a daily MBD of $-0.4 \%$ and NRMSD of $1 \%$ for Adelaide show an improvement in the model's performance. This is an important result because whilst a degree of difference at the five-minute level is understandable due to the synthetic nature of the method, it is desirable to have the hourly and daily totals comparing well. These results also compare favourable with Polo et al. (2011) which report a NRMSD of $15 \%$ for hourly and $2-4 \%$ for daily.

Also of interest is how the variation in the synthetically interpolated five-minute DNI compares with the variation in the observed. The Adelaide daily standard deviation MBD of $-0.05 \%$ and NRMSD of $4.2 \%$ indicate the synthetic is capturing the daily variation of the observed. More importantly, the five-minute standard deviations of the synthetic and observed compare very well with 390.0 and 391.3 respectively for Adelaide, and 373.5 and 372.4 respectively for Woomera.

Figures 4(a) and 4(b) show the scatter plots of the hourly irradiation totals from the synthetic DNI compared to the hourly irradiation totals from the observed DNI for Adelaide and Woomera respectively. Both plots indicate a high correlation between the synthetic and observed.

Whilst not shown here, the cumulative distribution functions (CDF) of the synthetically interpolated fiveminute DNI compares favourably to the CDF of the observed five-minute DNI for both Adelaide and Woomera. 
The overall results presented here indicate that this method of generating synthetically interpolated five-minute data is performing very well. Importantly the synthetic DNI is capturing the overall shape of the observed and obtaining very similar hourly irradiation totals. More importantly, the synthetic is capturing the same degree of variation to that found in the observed.

Of particular interest is how well this model is also performing for Woomera. This is an important result because it means the model could be applied to hourly satellite DNI data to extend the spatial coverage. The result being high resolution temporal and spatial DNI.

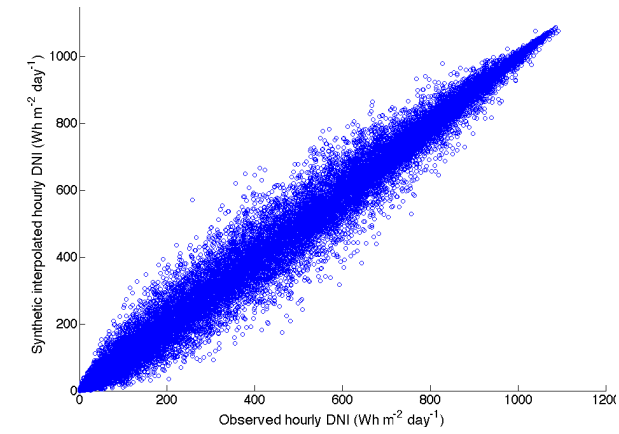

(a) Adelaide scatter plot

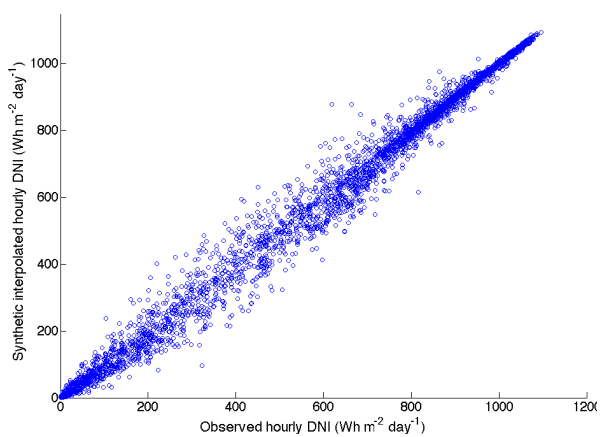

(b) Woomera scatter plot

\section{CONCLUSIONS}

Figure 4. Hourly direct normal irradiation scatter plots

The work presented here shows a method for generating synthetically interpolated five-minute DNI from hourly DNI. The method is still being developed but initial results show the shape, variation and statistical properties of the synthetic compare favourably to observed. Also, the results indicate the model, which is based on on five-minute DNI from Adelaide, could be applied to other locations where only hourly DNI exists.

However, there are still some aspects of this model that require further investigation.

The varying bias throughout the day could be because clouds behave differently in the morning than in the afternoon. A simple fix might include dividing the clear sky indexes into two groups; morning and afternoon.

Whilst bootstrapping from each clear sky index is used here, it may not be possible when there is a smaller amount of observed five-minute DNI to develop a model from. In this case, fitting a distribution to the categories will be required.

In order to determine the spatial coverage to which this model can be applied to hourly DNI, more comparisons against other spatially diverse locations are required.

\section{REFERENCES}

Assunção, H. F., J. F. Escobedo, and a. P. Oliveira (2007, February). A new algorithm to estimate sky condition based on 5 minutes-averaged values of clearness index and relative optical air mass. Theoretical and Applied Climatology 90(3-4), 235-248.

Boxel, J. V. (2002). Modelling Global Radiation for the Portofino area in Italy. Institute for Biodiversity and Ecosystem Dynamics IBED, University of Amsterdam, Netherlands.

Bureau of Meteorology (2013). About One Minute Solar Data.

Efron, B. (1992). Bootstrap Methods: Another Look at the Jackknife. In Breakthroughs in Statistics, pp. 569-593. Springer.

Gansler, R. A., S. A. Klein, and W. A. Beckman (1995). Investigation of minute solar radiation data. Solar Energy 55(1), 21-27.

Meyer, R., H. G. Beyer, J. Fanslau, N. Geuder, A. Hammer, T. Hirsch, C. Hoyer-klick, N. Schmidt, and M. Schwandt (2009). Towards Standardization Of CSP Yield Assessments. SolarPACES, Germany, 1-8.

Polo, J., L. Zarzalejo, R. Marchante, and A. Navarro (2011, May). A simple approach to the synthetic generation of solar irradiance time series with high temporal resolution. Solar Energy 85(5), 1164-1170.

Suehrcke, H. and P. G. McCormick (1988). The frequency distribution of instantaneous insolation values. Solar Energy 40(5), 413-422. 